九州大学学術情報リポジトリ

Kyushu University Institutional Repository

\title{
A STOCHASTIC APPROXIMATION WITH A SEQUENCE OF DEPENDENT RANDOM VARIABLES
}

Watanabe, Masafumi

Department of Applied Mathematics, Fukuoka University

https://doi.org/10.5109/13146

出版情報: 統計数理研究. 19 (3/4), pp. 25-42，1981-03. Research Association of Statistical Sciences

バージョン :

権利関係 : 


\title{
A STOCHASTIC APPROXIMATION WITH A SEQUENCE OF DEPENDENT RANDOM VARIABLES
}

\author{
By
}

\author{
Masafumi WatanABE
}

(Received September 20, 1980)

\begin{abstract}
Summary
Let $\left\{Y_{n}\right\}$ be a sequence of dependent random variables and $\left\{\Phi_{n}(\cdot, \cdot)\right\}$ be a sequence of Borel functions. Let $\theta_{n}$ be a solution of the equation $M_{n}(x)=0$ for each $n \geqq 1$, where $M_{n}(x)=\mathrm{E} \Phi_{n}\left(x, Y_{n}\right)$. A Robbins-Monro type stochastic approximation procedure $X_{n+1}$ $=X_{n}-a_{n} \Phi_{n}\left(X_{n}, Y_{n}\right)$ is considered for estimating $\theta_{n}$ for $n$ sufficiently large. Under some assumptions about $\left\{a_{n}\right\},\left\{\theta_{n}\right\},\left\{Y_{n}\right\}$ and $\left\{\Phi_{n}(\cdot, \cdot)\right\}$ which may not include the fundamental condition $\mathrm{E}\left[\Phi_{n}\left(X_{n}, Y_{n}\right) \mid X_{1}\right.$, $\left.\cdots, X_{n}\right]=M_{n}\left(X_{n}\right)$ a.s., the a.s. convergence and in mean-square convergence of $\left|X_{n}-\theta_{n}\right|$ to zero are studied.
\end{abstract}

\section{Introduction.}

This paper is a continuation of our previous paper [7] and is concerened with the following Robbins-Monro type stochastic approximation method with a sequence of dependent random variables.

Let $\left\{Y_{n}\right\}$ be a sequence of $\boldsymbol{R}^{M}$-valued random vectors and $\left\{\Phi_{n}(\cdot, \cdot)\right\}$ be a sequence of $\boldsymbol{R}^{N}$-valued Borel functions defined on $\boldsymbol{R}^{N} \times \boldsymbol{R}^{M}$. Assume that $\mathrm{E} \Phi_{n}\left(x, Y_{n}\right)$ exists for all $x \in \boldsymbol{R}^{N}$ and $n \geqq 1$, and it is unknown to us. Assuming that the equation

$$
\mathrm{E} \Phi_{n}\left(x, Y_{n}\right)=\mathbf{0} \quad\left(\mathbf{0} \text { denotes the zero vector of } \boldsymbol{R}^{N}\right)
$$

has a solution $x=\theta_{n}$ for each $n \geqq 1$, it is desired to estimate $\theta_{n}$ for $n$ sufficiently large on the basis observed values $\Phi_{1}\left(X_{1}, Y_{1}\right), \Phi_{2}\left(X_{2}, Y_{2}\right), \cdots$ at the points $\left(X_{1}, Y_{1}\right),\left(X_{2}, Y_{2}\right), \cdots$ where $X_{1}, X_{2}, \cdots$ are produced by the following recurrence relation;

$$
\left\{\begin{array}{l}
X_{1}=\text { an arbitrary constant vector of } \boldsymbol{R}^{N} \\
X_{n+1}=X_{n}-a_{n} \Phi_{n}\left(X_{n}, Y_{n}\right),
\end{array}\right.
$$

where $\left\{a_{n}\right\}$ is a decreasing sequence of positive numbers which converges to zero. The above situation will be concerned throughout this paper.

In the above problem, if the condition

Department of Applied Mathematics, Fukuoka University, Fukuoka 814-01, Japan 


$$
\mathrm{E}\left[\Phi_{n}\left(X_{n}, Y_{n}\right) \mid X_{1}, X_{2}, \cdots, X_{n}\right]=M_{n}\left(X_{n}\right) \text { a.s. }
$$

where $M_{n}(x)=\mathrm{E} \Phi_{n}\left(x, Y_{n}\right)$, is satisfied then this is considered as the usual RobbinsMonro stochastic approximation method. For example, if $\left\{Y_{n}\right\}$ is a sequence of independent random vectors then (1.3) is automatically satisfied under the procedure (1.2). Hence this is the case of the usual Robbins-Monro stochastic approximation. If $\left\{Y_{n}\right\}$ is a sequence of dependent random vectors then (1.3) may not be satisfied.

Thus, in this paper we shall study the above stochastic approximation under the situation that (1.3) does not hold. In [7], we considered the above problem under the assumption that $\Phi_{n}(x, y)$ can be expressed in the form of $\left(x-\theta_{n}\right) A_{n}(y)+\Gamma_{n}(y)$. And the a.s. convergence of the process (1.2) was investigated. In this paper we consider the same problem under some assumptions which are more general than ours in [7].

This paper consists of five sections. In Section 2 we give notation and four lemmas to be used throughout the paper. In Section 3 the conditions that have to impose on the procedure (1.2) are described. In Section 4 we shall give results about the a.s. convergence and in mean-square convergence of (1.2). In Section 5 we shall treat two examples of results. One of them was discussed in our previous paper [7].

\section{Notations and lemmas.}

The conventions introduced here hold throughout. Let $\boldsymbol{R}^{k}$ be $k$-dimensional Euclidian space. If $a, b \in \boldsymbol{R}^{N}$ and $c, d \in \boldsymbol{R}^{N_{0}},\langle a, b\rangle$ and $\langle c, d\rangle_{0}$ denote their inner products, respectively. The Euclidian norms of $a \in \boldsymbol{R}^{N}$ and $c \in \boldsymbol{R}^{N_{0}}$ are denoted by $|a|$ and $|c|_{0}$, respectively, of course, $|a|=\langle a, a\rangle^{1 / 2}$ and $|c|_{0}=\langle c, c\rangle_{0}^{1 / 2}$.

Let $(\Omega, \mathcal{A}, P)$ be a probability space. Define the dependent coefficient of $\sigma$-fields $\mathcal{A}^{\prime}$ and $\mathcal{A}^{\prime \prime}$ which are sub- $\sigma$-fields of $\mathcal{A}$ by the relation;

$$
\phi\left(\mathscr{A}^{\prime}, \mathscr{A}^{\prime \prime}\right)=\sup _{A \in \mathscr{A}^{\prime}}\left(\underset{\omega \in \Omega}{\operatorname{ess} \sup }\left|P\left(A \mid \mathcal{A}^{\prime}\right)(\omega)-P(A)\right|\right) .
$$

For the above definition of the dependent coefficient we refer to Iosifescu and Theodorescu [1]. Let $\mathcal{A}_{i}, i \in T$ be sub- $\sigma$-fields of $\mathcal{A}_{\text {. Then }} \bigvee_{i \in T} \mathcal{A}_{i}$ denotes the $\sigma$-fields generated by the join of the $\sigma$-fields $\mathcal{A}_{i}, i \in T$.

Next we shall give four lemmas to be used throughout the paper. The following Lemma 1 is given in [4].

LEMMA 1. Let $\left\{\alpha_{n}\right\}$ and $\left\{v_{n}\right\}$ be sequences of non-negative numbers such that

(i) $\quad \sum v_{n}<\infty, \quad \Sigma \alpha_{n}<\infty$.

If $\left\{x_{n}\right\}$ is a sequence of non-negative numbers such that, for some intege: $n_{0}$ and for all $n \geqq n_{0}$,

(ii) $x_{n+1} \leqq \max \left\{h,\left(1+\alpha_{n}\right) x_{n}+v_{n}\right\}$

where $h>0$, then $\left\{x_{n}\right\}$ is bounded.

Using Lemma 2.3 of [6], we obatin the following lemma.

LEMMA 2. Let $\left\{h_{n}\right\},\left\{a_{n}\right\},\left\{b_{n}\right\},\left\{d_{n}\right\},\left\{u_{n}\right\},\left\{v_{n}\right\}$ and $\left\{w_{n}\right\}$ be sequences $f$ rai numbers such that 
(i) $h_{n} \geqq 0, \quad \lim _{n \rightarrow \infty} h_{n}=0$

(ii) $\quad a_{n} \geqq a_{n+1} \geqq 0, \quad \lim _{n \rightarrow \infty} a_{n}=0, \quad \sum a_{n}=\infty$

(iii) $u_{n}>0, \sup _{n} u_{n}<\infty, \sup _{n}\left\{u_{n}\left|a_{n}^{-1}-a_{n+1}^{-1}\right|\right\}<\infty$

(iv) $\lim _{n \rightarrow \infty} b_{n}=0, \quad \sum\left|v_{n}\right|<\infty$

(v) $\lim _{n \rightarrow \infty} a_{n} u_{n}^{-1} \sum_{k=1}^{n} w_{k}=0$

(vi) $d_{n} \geqq 0, \quad \sum d_{n}<\infty$.

If $\left\{x_{n}\right\}$ is a sequance of non-negative numbers such that, for some positive integer $n_{0}$ and for all $n \geqq n_{0}$,

(vii) $x_{n+1} \leqq \max \left\{h_{n},\left(1-a_{n}+d_{n}\right) x_{n}+a_{n} b_{n}+a_{n} w_{n}+v_{n}\right\}$,

then it holds that $\lim _{n \rightarrow \infty} x_{n}=0$.

Proof. It involves no loss of generality to assume

$$
1-a_{n} \geqq \frac{1}{2} \quad \text { for all } n \geqq n_{0} .
$$

Let $N$ and $n$ be arbitrary positive integers such that $n_{0}<N<n$. Set

$$
y_{n}=a_{n} b_{n}+a_{n} w_{n}+v_{n} .
$$

The iterate (vii) back to $N$. This yields

$$
x_{n+1} \leqq \max \left\{\max _{N \leqq k \leqq n}\left\{T_{k+1}^{(n)} h_{k}+\sum_{i=k+1}^{n} T_{i+1}^{(n)} y_{j}\right\}, T_{N}^{(n)} x_{N}+\sum_{i=N}^{n} T_{i+1}^{(n)} y_{i}\right\}
$$

where

$$
T_{k}^{(n)}=\left\{\begin{array}{lll}
\prod_{j=k}^{n}\left(1-a_{j}+d_{j}\right) & \text { if } & 1 \leqq k \leqq n \\
1 & \text { if } & k=n+1
\end{array}\right.
$$

Hence we obtain

$$
x_{n+1} \leqq \max _{N \leqq k \leqq n}\left\{T_{k+1}^{(n)} h_{k}\right\}+T_{N}^{(n)} x_{N}+2 \max _{N \leq k \leq n}\left|\sum_{i=k}^{n} T_{i+1}^{(n)} y_{i}\right| .
$$

Now from (i) and (v), for any $\varepsilon>0$ we can chose $N$ so that

$$
\begin{aligned}
& \sup _{N \leqq n} h_{n}<\varepsilon, \\
& \sup _{N-1 \leqq n}\left|a_{n} u_{n}^{-1} \sum_{k=1}^{n} w_{k}\right|<\varepsilon .
\end{aligned}
$$


Since $1-a_{n}+d_{n}=\left(1-a_{n}\right)\left(1-\left(1-a_{n}\right)^{-1} d_{n}\right),(2.2)$ implies

$$
1-a_{n}+d_{n} \leqq\left(1-a_{n}\right)\left(1+2 d_{n}\right) \leqq 1+2 d_{n} .
$$

Hence, (vi) and (2.4) imply that there exists a positive constant $K_{1}=\prod_{n=1}^{\infty}\left(1+2 d_{n}\right)$ such that

$$
\max _{N \leqq k \leqq n} T_{k+1}^{(n)} h_{k}<K_{1} \varepsilon
$$

And it is easily seen from (ii) and (vi) that

$$
\lim _{n \rightarrow \infty} T_{N}^{(n)} x_{N} \leqq \lim _{n \rightarrow \infty} K_{1} x_{N} \prod_{k=N}^{n}\left(1-a_{k}\right)=0 .
$$

Note that

$$
\left|\sum_{i=k}^{n} T_{i+1}^{(n)} y_{i}\right| \leqq \sum_{i=n_{0}}^{n} T_{i+1}^{(n)}\left(a_{i}\left|b_{i}\right|+v_{i} \mid\right)+\left|\sum_{i=k}^{n} a_{i} w_{i} T_{i+1}^{(n)}\right| \cdot
$$

First, we shall prove that

$$
\lim _{n \rightarrow \infty} \sum_{i=n_{0}}^{n} T_{i+1}^{(n)}\left(a_{i}\left|b_{i}\right|+\left|v_{i}\right|\right)=0 .
$$

This is obtained with the aid of Lemma 2.3 of [6], which states that if $\left\{A_{n}\right\}$ is a sequence of non-negative numbers satisfying

$$
A_{n+1} \leqq\left(1-a_{n+1}\right) A_{n}+a_{n+1} b_{n+1}+v_{n+1}
$$

where $a_{n} \geqq 0, \lim _{n \rightarrow \infty} a_{n}=0, \sum a_{n}=\infty, b_{n} \geqq 0, \lim _{n \rightarrow \infty} b_{n}=0, v_{n} \geqq 0$ and $\sum v_{n}<\infty$, then $\lim _{n \rightarrow \infty} A_{n}=0$. Since $\left(1-a_{n}+d_{n}\right) \leqq\left(1-a_{n}\right)\left(1+2 d_{n}\right)$ and $\sum d_{n}<\infty$, it follows that

$$
\sum_{i=n_{0}}^{n} T_{i+1}^{(n)}\left(a_{i}\left|b_{i}\right|+\left|v_{i}\right|\right) \leqq K_{1} \sum_{i=n_{0}}^{n}\left(a_{i}\left|b_{i}\right|+\left|v_{i}\right|\right) \prod_{k=i+1}^{n}\left(1-a_{k}\right)
$$

where $K_{1}=\prod_{n=1}^{\infty}\left(1+2 d_{n}\right)<\infty$. Define $A_{n}=\sum_{i=n_{0}}^{n}\left(a_{i}\left|b_{i}\right|+\left|v_{i}\right|\right) \times \prod_{k=i+1}^{n}\left(1-a_{k}\right)$. Then Lemma 2.3 of [6] gives (2.9).

Next, let us define $u_{0}=a_{1}=1, s_{0}=0$ and for $n \geqq k \geqq 1$,

$$
s_{n}=a_{n} u_{n}^{-1} \sum_{k=1}^{n} w_{k}, \quad s_{k}^{(n)}=\sum_{i=k}^{n} a_{i} w_{i} T_{i+1}^{(n)} .
$$

Since $w_{i}=a_{i}^{-1} u_{i} s_{i}-a_{i-1}^{-1} u_{i-1} s_{i-1}$, we obtain

$$
\begin{aligned}
s_{k}^{(n)} & =u_{n} s_{n}-a_{k} a_{k-1}^{-1} u_{k-1} s_{k-1} T_{k+1}^{(n)}+\sum_{j=k}^{n-1} a_{j}^{-1} u_{j} s_{j}\left(a_{j} T_{j+1}^{(n)}-a_{j+1} T_{j+2}^{(n)}\right) \\
& =u_{n} s_{n}-a_{k} a_{k-1}^{-1} u_{k-1} s_{k-1} T_{k+1}^{(n)}+\sum_{j=k}^{n-1} a_{j+1} s_{j} u_{j} T_{j+2}^{(n)}\left(a_{j+1}^{-1}-a_{j}^{-1}-1\right) .
\end{aligned}
$$

Therefore it follows from (ii), (iii) and (vi) that there exist positive constants $K_{2}$ and $K_{3}$ such that 


$$
\left|s_{k}^{(n)}\right| \leqq K_{2} \sup _{N-1 \leqq n}\left|s_{n}\right|+K_{3} \sum_{j=n_{0}}^{n} a_{j}\left|s_{j-1}\right| T_{j+1}^{(n)} .
$$

Applying Lemma 2.3 of $[6]$ to the second term in the righthand side of (2.10) we obtain

$$
\lim _{n \rightarrow \infty} \sum_{j=n_{0}}^{n} a_{j}\left|s_{j-1}\right| T_{j+1}^{(n)}=0 .
$$

Therefore (2.3) and (2.5) to (2.11) imply

$$
\limsup _{n \rightarrow \infty} x_{n} \leqq\left(K_{1}+2 K_{2}\right) \varepsilon \text {. }
$$

Because $\varepsilon$ is arbitrary, this concludes the proof of Lemma 2 .

REMARK. Examples of two sequences $\left\{a_{n}\right\}$ and $\left\{u_{n}\right\}$ satisfying (ii) and (iii) are easy to obtain. For example, $a_{n}=n^{-\alpha} \quad(0<\alpha \leqq 1)$ and $u_{n}=n^{-\beta}(0 \leqq \beta)$ satisfy (ii) and (iii). And $a_{n}=(n \log n)^{-1}$ and $u_{n}=(\log n)^{-1}$ satisfy (ii) and (iii) also.

Finally we state without proof two lemmas which are proved in [1].

LEMMA 3. (The strong law of large numbers). Let $\left\{X_{n}\right\}$ be a sequence of random variables. Suppose that there exist a positive integer $n_{0}$ and a decreasing sequence of positive numbers $\left\{a_{n}\right\}$ such that

(i) $\limsup _{n \rightarrow \infty} \phi\left(\underset{1 \leqq i \leqq n}{\bigvee} \mathcal{A}_{i}, \underset{n+n_{0} \leqq i}{\bigvee} \mathcal{A}_{i}\right)<1$,

(ii) $\sum_{n=1}^{\infty}\left\{\sup _{m} \phi\left(\mathcal{A}_{m}, \mathcal{A}_{m+n}\right)\right\}^{1 / 2}<\infty$,

(iii) $\lim _{n \rightarrow \infty} a_{n}=0$,

(iv) $\sum_{n=1}^{\infty} a_{n}^{2} \mathrm{E}\left(X_{n}-\mathrm{E} X_{n}\right)^{2}<\infty$,

where $\mathcal{A}_{i}$ is the $\sigma$-field generated by $X_{i}$ and $\phi(\cdot, \cdot)$ is defined by (2.1). Then it holds that

$$
\lim _{n \rightarrow \infty} a_{n} \sum_{i=1}^{n}\left(X_{i}-\mathrm{E} X_{i}\right)=0 \quad \text { a.s. . }
$$

REMARK. Let $\left\{X_{n}\right\}$ be a sequence of $\boldsymbol{R}^{N}$-valued random vectors satisfying (i) and (ii). And (iii) and

(iv)' $\sum a_{n}^{2} \mathrm{E}\left|X_{n}-\mathrm{E} X_{n}\right|^{2}<\infty$ (" $|\cdot|$ " denotes the Euclidian norm) are satisfied. Then it also holds that

$$
\lim _{n \rightarrow \infty} a_{n}\left|\sum_{i=1}^{n}\left(X_{i}-\mathrm{E} X_{i}\right)\right|=0 \quad \text { a.s. . }
$$

LEMMA 4. Let the random variable $X_{i}$ be $A_{i}$-measurable for $i=1,2$. Suppose that $\mathrm{E} X_{i}^{2}<\infty, i=1,2$. Then

$$
\left|\mathrm{E} X_{1} X_{2}-\mathrm{E} X_{1} \mathrm{E} X_{2}\right| \leqq 2 \phi^{1 / 2}\left(\mathcal{A}_{1}, \mathcal{A}_{2}\right)\left(\mathrm{E} X_{1}^{2}\right)^{1 / 2}\left(\mathrm{E} X_{2}^{2}\right)^{1 / 2} .
$$


REMARK. Let $X_{i}, i=1,2$ be $\boldsymbol{R}^{N}$-valued random vectors satisfying $\mathrm{E}\left|X_{i}\right|^{2}<\infty$, $i=1,2$. And $X_{i}$ is $A_{i}$-measurable for $i=1,2$. Then it is easily seen that

$$
\left|\mathrm{E}\left\langle X_{1}, X_{2}\right\rangle-\left\langle\mathrm{E} X_{1}, \mathrm{E} X_{2}\right\rangle\right| \leqq 2 \phi^{1 / 2}\left(\mathcal{A}_{1}, \mathcal{A}_{2}\right)\left(\mathrm{E}\left|X_{1}\right|^{2}\right)^{1 / 2}\left(\mathrm{E}\left|X_{2}\right|^{2}\right)^{1 / 2} .
$$

\section{Assumptions.}

Assume that, for each $n \geqq 1$, the equation (1.1) has a solution $\theta_{n}$. Let $\mathcal{A}_{n}$ be the $\sigma$-field generated by the $\boldsymbol{R}^{M}$-valued random vector $Y_{n}$ for each $n \geqq 1$. Let $\left\{a_{n}\right\}$ be the decreasing sequence of positive numbers which is used in the procedure (1.2). And $\left\{a_{n}\right\}$ satisfies the following conditions $\mathrm{A} 1$ and $\mathrm{A} 2$.

A1: $\lim _{n \rightarrow \infty} a_{n}=0, \quad \sum a_{n}=\infty$.

A2: There exists a non-increasing sequence of positive numbers $\left\{\hat{o}_{n}\right\}$ such that

$$
\lim _{n \rightarrow \infty} \delta_{n}=0, \quad \sum a_{n} \delta_{n}<\infty
$$

Moreover we shall make the following assumptions about $\left\{a_{n}\right\}$ and $\left\{\delta_{n}\right\}$.

A3: $\quad a_{n} \delta_{n}^{-3}>a_{n+1} \hat{\delta}_{n+1}^{-3}, \quad \lim _{n \rightarrow \infty} a_{n} \delta_{n}^{-3}=0$.

A4: $\sup _{n}\left|a_{n}^{-1}-a_{n+1}^{-1}\right|<\infty$.

A5: $\sum a_{n}^{2} \bar{\delta}_{n}^{-2}<\infty$.

Next we shall make the following assumptions B1 to B7 which are used in the proof of Theorem 1 .

B1: For each $n \geqq 1$, let $f_{n}(\cdot, \cdot)$ be a real valued Borel function defined on $\boldsymbol{R}^{v} \times \boldsymbol{R}^{M}$

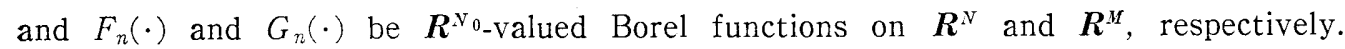
Suppose that there exist a sequence of non-negative numbers $\left\{\varepsilon_{n}\right\}$ which converges to zero and a positive constant $\alpha_{0}$ such that, for all $y \in \boldsymbol{R}^{M}$ and for all $n \geqq 1$,

$$
\left\langle x-\theta_{n}, \Phi_{n}(x, y)\right\rangle \geqq \max \left\{f_{n}(x, y), \alpha_{0}\left|x-\theta_{n}\right|^{2}+\left\langle F_{n}(x), G_{n}(y)\right\rangle_{0}\right\}
$$

if $\left|x-\theta_{n}\right|^{2}>\varepsilon_{n}$.

B2: There exist sequences of non-negative Borel functions $\left\{g_{n}(\cdot)\right\}$ and $\left\{h_{n}(\cdot)\right\}$ defined on $\boldsymbol{R}^{M}$ such that

(i) $\left|f_{n}(x, y)\right| \leqq g_{n}(y)\left|x-\theta_{n}\right|^{2}+h_{n}(y)$

for all $x \in \boldsymbol{R}^{N}, y \in \boldsymbol{R}^{M}$ and $n \geqq 1$, where $f_{n}(\cdot, \cdot)$ is to be given in B1,

(ii) $\sum a_{n} \mathrm{E} g_{n}\left(Y_{n}\right)<\infty$,

(iii) $\sum a_{n} \delta_{n}^{2} \mathrm{E} h_{n}\left(Y_{n}\right)<\infty$.

B3: Let $\left\{F_{n}(\cdot)\right\}$ be as given in B1. There exist non-negative constants $K_{i}$, $1 \leqq i \leqq 4$ and three sequences of non-negative numbers $\left.\left\{b_{n}\right\}, i c_{n}\right\}$ and $\left\{d_{n}\right\}$ such that 
(i) $\left|F_{n}(x)\right|_{0} \leqq K_{1}\left|x-\theta_{n}\right|^{2}+K_{2}\left|x-\theta_{n}\right|+K_{3} \quad$ for all $x \in \boldsymbol{R}^{N}$ and $n \geqq 1$,

(ii) $\left|F_{n}(x)-F_{n}\left(x^{\prime}\right)\right|_{0} \leqq K_{4}\left(\left|x-\theta_{n}\right|+\left|x^{\prime}-\theta_{n}\right|+1\right)\left|x-x^{\prime}\right|$

for all $x, x^{\prime} \equiv \boldsymbol{R}^{v}$ and $n \geqq 1$,

(iii) $\left|F_{n}(x)-F_{n+1}(x)\right|_{0} \leqq b_{n}\left|x-\theta_{n}\right|^{2}+c_{n}\left|x-\theta_{n}\right|+d_{n} \quad$ for all $x \in \boldsymbol{R}^{N}$ and $n \geqq 1$,

(iv) $\sum b_{n} \delta_{n}<\infty$,

(v) $\sup _{n} c_{n} \delta_{n}<\infty, \quad \sum c_{n} \dot{\delta}_{n}^{2}<\infty$,

(vi) $\sum d_{n} \delta_{n}^{3}<\infty$.

B4: Let $\left\{G_{n}(\cdot)\right\}$ be as given in B1.

(i) $\lim _{n \rightarrow \infty}\left|\mathrm{E} G_{n}\left(Y_{n}\right)\right|_{0}=0$,

(ii) $\sum a_{n}^{2} \delta_{n}^{-6} \mathrm{E}_{i} G_{n}\left(Y_{n}\right)-\left.\mathrm{E} G_{n}\left(Y_{n}\right)\right|_{0} ^{2}<\infty$.

B5: There exist sequences of non-negative Borel functions $\left\{\alpha_{n}(\cdot)\right\}$ and $\left\{\beta_{n}(\cdot)\right\}$ defined on $\boldsymbol{R}^{u}$ such that

(i) $\left|\Phi_{n}(x, y)\right|^{2} \leqq \alpha_{n}(y)\left|x-\theta_{n}\right|^{2}+\beta_{n}(y) \quad$ for all $x \in \boldsymbol{R}^{v}, y \in \boldsymbol{R}^{n}$ and $n \geqq 1$,

(ii) $\sum a_{n} \delta_{n} \mathrm{E} \alpha_{n}\left(Y_{n}\right)<\infty$,

(iii) $\sum a_{n} \hat{\partial}_{n}^{3} \mathrm{E} \beta_{n}\left(Y_{n}\right)<\infty$.

B6: Let $n_{0}$ be a positive integer.

(i) $\limsup _{n \rightarrow \infty} \phi\left(\underset{1 \leqq i \leq n}{\bigvee} \mathcal{A}_{i}, \underset{n+n_{0} \leqq i}{\bigvee} A_{i}\right)<1$,

(ii) $\sum_{n=1}^{\infty} \sup _{m} \phi^{1 / 2}\left(\mathcal{A}_{m}, \mathcal{A}_{m+n}\right)<\infty$.

B7 : $\lim _{n \rightarrow \infty} a_{n}^{-1}\left|\theta_{n}-\theta_{n+1}\right|=0$.

Finally we shall make the following assumptions $\mathrm{C} 1$ to $\mathrm{C} 3$ which are used in the proof of Theorem 2 .

C1: There exist a positive constant $\alpha_{0}$ and a sequence of $\boldsymbol{R}^{v}$-valued Borel functions $\left\{\gamma_{n}(\cdot)\right\}$ defined on $\boldsymbol{R}^{M}$ such that

(i) $\left\langle x-\theta_{n}, \Phi_{n}(x, y)\right\rangle \geqq \alpha_{0}\left|x-\theta_{n}\right|^{2}+\left\langle x-\theta_{n}, \gamma_{n}(y)\right\rangle$

for all $x \in \boldsymbol{R}^{N}, y \in \boldsymbol{R}^{N}$ and $n \geqq 1$,

(ii) $\lim _{n \rightarrow \infty}\left|\mathrm{E} \gamma_{n}\left(\dot{Y}_{n}\right)\right|=0$,

(iii) $\sup _{n} \mathrm{E}\left|\gamma_{n}\left(Y_{n}\right)-\mathrm{E} \gamma_{n}\left(Y_{n}\right)\right|^{2}<\infty$.

C2: There exist a positive constant $\alpha_{1}$ and a sequence of non-negative Borel functions $\left\{\beta_{n}(\cdot)\right\}$ defined on $\boldsymbol{R}^{M}$ such that 
(i) $\left|\Phi_{n}(x, y)\right|^{2} \leqq \alpha_{1}\left|x-\theta_{n}\right|^{2}+\beta_{n}(y) \quad$ for all $x \in \boldsymbol{R}^{N}, y \in \boldsymbol{R}^{M}$ and $n \geqq 1$,

(ii) $\sup _{n} \mathrm{E}_{3} \beta_{n}\left(Y_{n}\right)<\infty$.

C3: $\sum_{n=1}^{\infty} \sup _{m} \phi^{1 / 2}\left(\underset{1 \leq i \leq m}{\bigvee} A_{i}, \underset{m+n \leq i}{\bigvee} A_{i}\right)<\infty$.

REMARKS. (1) Since $\left\{\delta_{n}\right\}$ is non-increasing and bounded, A3 implies

$\mathrm{A}^{\prime}: \quad a_{n} \hat{o}_{n}^{-1}>a_{n+1} \delta_{n+1}^{-1}, \quad \lim _{n \rightarrow \infty} a_{n} \delta_{n}^{-1}=0$.

Because $a_{n}^{2}=a_{n} \delta_{n} a_{n} \delta_{n}^{-1}, \mathrm{~A} 3^{\prime}$ and B5(ii) imply

$$
\sum a_{n}^{2} \alpha_{n}\left(Y_{n}\right)<\infty \quad \text { a.s. . }
$$

And because $a_{n}^{2}=a_{n} \delta_{n}^{3} a_{n} \delta_{n}^{-3}, \mathrm{~A} 3$ and B5(iii) imply

$$
\sum a_{n}^{2} \beta_{n}\left(Y_{n}\right)<\infty \text { a.s. . }
$$

(2) Using the inequality $2 a b \leqq k a^{2}+k^{-1} b^{2}$ which holds for and $k>0$, it follows from $B 3(i)$ that

$$
\begin{aligned}
& \alpha_{0}\left|x-\theta_{n}\right|^{2}+\left\langle F_{n}(x), G_{n}(y)\right\rangle_{0} \\
\leqq & \frac{\delta_{n}}{2}\left|x-\theta_{n}\right|^{2}+\frac{\delta_{n}^{-1} \alpha_{0}^{2}}{2}+K_{1}\left|G_{n}(y)\right|_{0}\left|x-\theta_{n}\right|^{2} \\
& +\frac{\delta_{n}}{2}\left|x-\theta_{n}\right|^{2}+\frac{\delta_{n}^{-1} K_{2}^{2}}{2}\left|G_{n}(y)\right|_{0}^{2}+K_{3}\left|G_{n}(y)\right|_{0} \\
= & \left(\delta_{n}+K_{1}\left|G_{n}(y)\right|_{0}\right)\left|x-\theta_{n}\right|^{2}+\frac{\delta_{n}^{-1} \alpha_{0}^{2}}{2}+\frac{\delta_{n}^{-1} K_{2}^{2}}{2}\left|G_{n}(y)\right|_{0}^{2}+K_{3}\left|G_{n}(y)\right|_{0} .
\end{aligned}
$$

If $\mathrm{A} 2$,

$$
\sum a_{n} \mathrm{E}\left|G_{n}\left(Y_{n}\right)\right|_{0}<\infty
$$

and

$$
\sum a_{n} \delta_{n} \mathrm{E}\left|G_{n}\left(Y_{n}\right)\right|_{0}^{2}<\infty
$$

are satisfied then we can chose $f_{n}(\cdot, \cdot)$ so that

$$
f_{n}(x, y)=\alpha_{0}\left|x-\theta_{n}\right|^{2}+\left\langle F_{n}(x), G_{n}(y)\right\rangle_{0} .
$$

Putting $g_{n}(y)=\delta_{n}+K_{1}\left|G_{n}(y)\right|_{0}$ and $h_{n}(y)=\frac{\delta_{n}^{-1} \alpha_{0}^{2}}{2}+\frac{\delta_{n}^{-1} K_{2}^{2}}{2}\left|G_{n}(y)\right|_{0}^{2}+K_{3}\left|G_{n}(y)\right|_{0}, \quad \mathrm{~A} 2$, (3.3) and (3.4) imply B2. If $a_{n} \delta_{n} \leqq a_{n}^{2} \delta_{n}^{-6}$ follows then B4(ii) implies (3.4), implying B2(iii). But B4 does not yield (3.3). Hence B2(ii) may not be satisfied. Thus it is needed to introduce the function $f_{n}(\cdot, \cdot)$ when $K_{1}>0$. If we can assume that $K_{1}=0$ then $f_{n}(\cdot, \cdot)$ is not needed.

(3) Since $\phi\left(\mathcal{A}_{m}, \mathcal{A}_{m+n}\right) \leqq \phi\left(\underset{1 \leqq i \leqq m}{\bigvee} \mathcal{A}_{i}, \underset{m+n \leqq i}{\bigvee} \mathscr{A}_{i}\right)$, the mixing condition C3 implies B6.

(4) Examples of two sequences $\left\{a_{n}\right\}$ and $\left\{\delta_{n}\right\}$ satisfying A1 to A4 are easy to 
obtain. For example, $a_{n}=n^{-\alpha}\left(\frac{7}{8}<\alpha \leqq 1\right)$ and $\delta_{n}=n^{-\beta}\left(1-\alpha<\beta \leqq \frac{\alpha}{7}\right)$ satisfy A1 to A4. And A5 is also satisfied. $a_{n}=n^{-\alpha^{\prime}}\left(\frac{3}{4}<\alpha^{\prime} \leqq 1\right)$ and $\delta_{n}=n^{-\beta^{\prime}}\left(1-\alpha^{\prime}<\beta^{\prime} \leqq \frac{\alpha^{\prime}}{3}\right)$ satisfy $\mathrm{A} 1, \mathrm{~A} 2, \mathrm{~A} 3^{\prime}, \mathrm{A} 4$ and $\mathrm{A} 5$, where $\mathrm{A} 3^{\prime}$ is to be given in (1).

\section{Results.}

Using Lemma 1 we obtain the following lemma.

Lemma 5. Let $X_{1}, X_{2}, \cdots$ be the Robbins-Monro type process defines by (1.2). Suppose that $\mathrm{A} 1$ to $\mathrm{A} 3, \mathrm{~B} 1, \mathrm{~B} 2, \mathrm{~B} 5$ and $\mathrm{B} 7$ are satisfied. Then it holds that there exists a nonnegative random variable $Z$ such that, for all $n \geqq 1$,

$$
\left|X_{n}-\theta_{n}\right| \leqq \delta_{n}^{-1} Z \text { a.s. . }
$$

Proof. We reduce the lemma to an a.s. pointwise application of Lemma 1. By virture of (1.2) we obtain directly

$$
\begin{aligned}
\left|X_{n+1}-\theta_{n+1}\right|^{2}= & \left|X_{n}-\theta_{n}\right|^{2}+a_{n}^{2}\left|\Phi_{n}\left(X_{n}, Y_{n}\right)\right|^{2}+\left|\theta_{n}-\theta_{n+1}\right|^{2} \\
& +2\left\langle X_{n}-\theta_{n}, \theta_{n}-\theta_{n+1}\right\rangle-2 a_{n}\left\langle\Phi_{n}\left(X_{n}, Y_{n}\right), \theta_{n}-\theta_{n+1}\right\rangle \\
& -2 a_{n}\left\langle X_{n}-\theta_{n}, \Phi_{n}\left(X_{n}, Y_{n}\right)\right\rangle .
\end{aligned}
$$

Using the inequality $2 a b \leqq k a^{2}+k^{-1} b^{2}(k>0)$ we obtain

$$
2\left|\left\langle X_{n}-\theta_{n}, \theta_{n}-\theta_{n+1}\right\rangle\right| \leqq a_{n} \delta_{n}\left|X_{n}-\theta_{n}\right|^{2}+a_{n} \delta_{n}^{-1} A_{n}
$$

where

$$
A_{n}=\left(a_{n}^{-1}\left|\theta_{n}-\theta_{n+1}\right|\right)^{2} .
$$

From B5(i) we botain

$$
\begin{aligned}
2 a_{n}\left|\left\langle\Phi_{n}\left(X_{n}, Y_{n}\right), \theta_{n}-\theta_{n+1}\right\rangle\right| \leqq & a_{n}^{2} \alpha_{n}\left(Y_{n}\right)\left|X_{n}-\theta_{n}\right|^{2} \\
& +a_{n}^{2} \beta_{n}\left(Y_{n}\right)+a_{n}^{2} A_{n},
\end{aligned}
$$

where $A_{n}$ is defined by (4.4). And from B1 and B2(i), if $\left|X_{n}-\theta_{n}\right|^{2}>\varepsilon_{n}$, then it follows that

$$
\begin{aligned}
-2 a_{n}\left\langle X_{n}-\theta_{n}, \Phi_{n}\left(X_{n}, Y_{n}\right)\right\rangle & \leqq 2 a_{n}\left|f_{n}\left(X_{n}, Y_{n}\right)\right| \\
& \leqq 2 a_{n} g_{n}\left(Y_{n}\right)\left|X_{n}-\theta_{n}\right|^{2}+2 a_{n} h_{n}\left(Y_{n}\right)^{2} .
\end{aligned}
$$

Substituting (4.3), (4.5), (4.6) and B5(i) into (4.2), if $\left|X_{n}-\theta_{n}\right|^{2}>\varepsilon_{n}$ then it follows that

$$
\begin{aligned}
\left|X_{n+1}-\theta_{n+1}\right|^{2} \leqq & \left(1+a_{n} \delta_{n}+2 a_{n}^{2} \alpha_{n}\left(Y_{n}\right)+2 a_{n} g_{n}\left(Y_{n}\right)\right)\left|X_{n}-\theta_{n}\right|^{2} \\
& +2 a_{n}^{2} \beta_{n}\left(Y_{n}\right)+2 a_{n} h_{n}\left(Y_{n}\right)+\left(2 a_{n}^{2}+a_{n} \delta_{n}^{-1}\right) A_{n} .
\end{aligned}
$$

Next we suppose that $\left|X_{n}-\theta_{n}\right|^{2} \leqq \varepsilon_{n}$. Substituting B5(i) into (4.2) we obtain

$$
\begin{aligned}
&\left|X_{n+1}+\theta_{n+1}\right|^{2} \leqq 3\left|X_{n}-\theta_{n}\right|^{2}+3 a_{n}^{2}\left|\Phi_{n}\left(X_{n}, Y_{n}\right)\right|^{2}+3\left|\theta_{n}-\theta_{n+1}\right|^{2} \\
& \leqq 3\left(1+a_{n}^{2} \alpha_{n}\left(Y_{n}\right)\right) \varepsilon_{n}+3 a_{n}^{2} \beta_{n}\left(Y_{n}\right)+3 a_{n}^{2} A_{n} \\
& \text { if }\left|X_{n}-\theta_{n}\right|^{2} \leqq \varepsilon_{n} .
\end{aligned}
$$


Since $\left\{\delta_{n}\right\}$ is non-increasing, (4.7) and (4.8) imply

where

$$
\delta_{n+1}^{2}\left|X_{n+1}-\theta_{n+1}\right|^{2} \leqq \max \left\{\delta_{n}^{2} h_{n},\left(1+d_{n}^{\prime}\right) \delta_{n}^{2}\left|X_{n}-\theta_{n}\right|^{2}+\delta_{n}^{2} v_{n}^{\prime}\right\}
$$

$$
\begin{aligned}
& h_{n}=3\left(1+a_{n}^{2} \alpha_{n}\left(Y_{n}\right)\right) \varepsilon_{n}+3 a_{n}^{2} \beta_{n}\left(Y_{n}\right)+3 a_{n}^{2}\left(a_{n}^{-1}\left|\theta_{n}-\theta_{n+1}\right|\right)^{2}, \\
& d_{n}^{\prime}=a_{n} \delta_{n}+2 a_{n}^{2} \alpha_{n}\left(Y_{n}\right)+2 a_{n} g_{n}\left(Y_{n}\right), \\
& v_{n}^{\prime}=2 a_{n}^{2} \beta_{n}\left(Y_{n}\right)+2 a_{n} h_{n}\left(Y_{n}\right)+\left(2 a_{n}^{2}+a_{n} \delta_{n}^{-1}\right)\left(a_{n}^{-1}\left|\theta_{n}-\theta_{n+1}\right|\right)^{2} .
\end{aligned}
$$

From (3.1), (3.2) and B7, if follows that

$$
\lim _{n \rightarrow \infty} h_{n}=0 \quad \text { a.s. . }
$$

From A2, (3.1) and B2(ii), it follows that

$$
\sum d_{n}^{\prime}<\infty \quad \text { a.s. . }
$$

And from (3.2), B2(iii), A2 and B7, it follows that

$$
\sum \delta_{n}^{2} v_{n}^{\prime}<\text { a.s. . }
$$

Therefore, using Lemma 1, (4.1) follows.

Using Lemma 3 and Lemma 5 we obtain the following lemma.

LEMMA 6. Suppose that the hypotheses of Lemma 5 are satisfied. Moreover, suppose that B3, B4(ii) and B6 are satisfied. Then it holds that

$$
\lim _{n \rightarrow \infty} a_{n} \sum_{k=1}^{n}\left\langle F_{k}\left(X_{k}\right), G_{k}\left(Y_{k}\right)-\mathrm{E} G_{k}\left(Y_{k}\right)\right\rangle_{0}=0 \quad \text { a.s. . }
$$

Proof. Define $\delta_{0}=a_{0}=1, S_{0}=\mathbf{0}$ (the zero vector of $\boldsymbol{R}^{N_{0}}$ ) and

$$
S_{n}=a_{n} \bar{\partial}_{n}^{-3} \sum_{k=1}^{n}\left(G_{k}\left(Y_{k}\right)-\mathrm{E} G_{k}\left(Y_{k}\right)\right) .
$$

Using Lemma 3 (The strong law of large numbers), it follows from A3 and B4(ii) that

$$
\lim _{n \rightarrow \infty}\left|S_{n}\right|_{0}=0 \text { a.s. . }
$$

Let us put

$$
a_{n} \sum_{k=1}^{n}\left\langle F_{k}\left(X_{k}\right), G_{k}\left(Y_{k}\right)-\mathrm{E} G_{k}\left(Y_{k}\right)\right\rangle_{0}=W_{n} .
$$

Since $G_{k}\left(Y_{k}\right)-\mathrm{E} G_{k}\left(Y_{k}\right)=a_{k}^{-1} \delta_{k}^{3} S_{k}-a_{k-1}^{-1} \delta_{k-1}^{3} S_{k-1}, W_{n}$ is rewritten

$$
W_{n}=\delta_{n}^{3}\left\langle F_{n}\left(X_{n}\right), S_{n}\right\rangle_{0}+a_{n} \sum_{j=1}^{n-1} a_{j}^{-1} \delta_{j}^{3}\left\langle F_{j}\left(X_{j}\right)-F_{j+1}\left(X_{j+1}\right), S_{j}\right\rangle_{0} .
$$

Then we obtain

$$
\begin{aligned}
\left|W_{n}\right| \leqq & \delta_{n}^{3}\left|F_{n}\left(X_{n}\right)\right|_{0}\left|S_{n}\right|_{0}+a_{n} \sum_{j=1}^{n} a_{j}^{-1} \delta_{j}^{3}\left|F_{j}\left(X_{j}\right)-F_{j}\left(X_{j+1}\right)\right|_{0}\left|S_{j}\right|_{0} \\
& +a_{n} \sum_{j=1}^{n} a_{j}^{-1} \delta_{j}^{3}\left|F_{j}\left(X_{j+1}\right)-F_{j+1}\left(X_{j+1}\right)\right|_{0}\left|S_{j}\right|_{0} .
\end{aligned}
$$


Hence, in order to show (4.11) we have to prove the following (a) to (c).
(a): $\lim _{n \rightarrow \infty} \delta_{n}^{3}\left|F_{n}\left(X_{n}\right)\right|_{0}\left|S_{n}\right|_{0}=0 \quad$ a.s. .
(b): $\lim _{n \rightarrow \infty} a_{n} \sum_{j=1}^{n} a_{j}^{-1} \hat{o}_{j}^{3}\left|F_{j}\left(X_{j}\right)-F_{j}\left(X_{j+1}\right)\right|_{0}\left|S_{j}\right|_{0}=0 \quad$ a.s. .
(c): $\lim _{n \rightarrow \infty} a_{n} \sum_{j=1}^{n} a_{j}^{-1} \hat{\delta}_{j}^{3}\left|F_{j}\left(X_{j+1}\right)-F_{j+1}\left(X_{j+1}\right)\right|_{0}\left|S_{j}\right|_{0}=0 \quad$ a.s. .

Proof OF (a). It is easily seen from B3(i), Lemma 5 and (4.12) that (a) holds.

PROOF OF (b). By virture of (1.2) and B5(i), we obtain

$$
\left|X_{j}-X_{j+1}\right|^{2} \leqq a_{j}^{2} \alpha_{j}\left(Y_{j}\right)\left|X_{j}-\theta_{j}\right|^{2}+a_{j}^{2} \beta_{j}\left(Y_{j}\right) .
$$

From B3(ii), (4.13) and Lemma 5 we obtain

$$
\begin{aligned}
\left|F_{j}\left(X_{j}\right)-F_{j}\left(X_{j+1}\right)\right|_{0} \leqq & K_{4}\left(2\left|X_{j}-\theta_{j}\right|+\left|X_{j}-X_{j+1}\right|+1\right)\left|X_{j}-X_{j+1}\right| \\
\leqq & 2 K_{4}\left(a_{j} \delta_{j}^{-2} \alpha_{j}^{1 / 2}\left(Y_{j}\right) Z^{2}+a_{j} \delta_{j}^{-1} \beta_{j}^{1 / 2}\left(Y_{j}\right) Z\right) \\
& +K_{4}\left(a_{j}^{2} \delta_{j}^{-2} \alpha_{j}\left(Y_{j}\right) Z^{2}+a_{j}^{2} \beta_{j}\left(Y_{j}\right)\right) \\
& +K_{4}\left(a_{j} \delta_{j}^{-1} \alpha_{j}^{1 / 2}\left(Y_{j}\right) Z+a_{j} \beta_{j}^{1 / 2}\left(Y_{j}\right)\right) \quad \text { a.s. }
\end{aligned}
$$

Define $Z_{0}=\max \left\{Z^{2}, Z, 1\right\}$. Noting $\lim _{n \rightarrow \infty} a_{n}=0$ and from A2 and A3, there exists a positive constant $K_{5}$ such that

$$
\begin{aligned}
\left|F_{j}\left(X_{j}\right)-F_{j}\left(X_{j+1}\right)\right|_{0} \leqq & K_{5} Z_{0}\left\{a_{j} \delta_{j}^{-2}\left(\alpha_{j}^{1 / 2}\left(Y_{j}\right)+\alpha_{j}\left(Y_{j}\right)\right)\right. \\
& \left.+a_{j} \delta_{j}^{-1}\left(\beta_{j}^{1 / 2}\left(Y_{j}\right)+\beta_{j}\left(Y_{j}\right)\right)\right\} \quad \text { a.s. . }
\end{aligned}
$$

Using Schwarz inequality, it follows from A2 and B5(ii) that

$$
\sum a_{n} \delta_{n} \alpha_{n}^{1 / 2}\left(Y_{n}\right) \leqq\left(\sum a_{n} \delta_{n}\right)^{1 / 2}\left(\sum a_{n} \delta_{n} \alpha_{n}\left(Y_{n}\right)\right)^{1 / 2}<\infty \quad \text { a.s. . }
$$

And it also follows from B5(iii) that

$$
\sum a_{n} \delta_{n}^{2} \beta_{n}^{1 / 2}\left(Y_{n}\right) \leqq\left(\sum a_{n} \delta_{n}\right)^{1 / 2}\left(\sum a_{n} \delta_{n}^{3} \beta_{n}\left(Y_{n}\right)\right)^{1 / 2}<\infty \quad \text { a.s. . }
$$

Hence it follows from (4.12), (4.14), (4.15), B5(ii) and B5(iii) that

$$
\sum \delta_{n}^{3}\left|F_{n}\left(X_{n}\right)-F_{n}\left(X_{n+1}\right)\right|_{0}\left|S_{n}\right|_{0}<\infty \quad \text { a.s. . }
$$

Therefore, from Kronecker lemma (see, e.g., [2], page 238), (b) follows.

Proof of (c). From B3(iii), (4.13) and Lemma 5 it follows that

$$
\begin{aligned}
\left|F_{j}\left(X_{j+1}\right)-F_{j+1}\left(X_{j+1}\right)\right|_{0} \leqq & 2 b_{j}\left|X_{j+1}-X_{j}\right|^{2}+2 b_{j}\left|X_{j}-\theta_{j}\right|^{2} \\
& +c_{j}\left|X_{j}-X_{j+1}\right|+c_{j}\left|X_{j}-\theta_{j}\right|+d_{j} \\
\leqq & 2 b_{j} \delta_{j}^{-2}\left(a_{j}^{2} \alpha_{j}\left(Y_{j}\right)+1\right) Z^{2}+2 b_{j} a_{j}^{2} \beta_{j}\left(Y_{j}\right) \\
& +c_{j} \delta_{j}^{-1}\left(a_{j} \alpha_{j}^{1 / 2}\left(Y_{j}\right)+1\right) Z \\
& +c_{j} a_{j} \beta_{j}^{1 / 2}\left(Y_{j}\right)+d_{j} \text { a.s. }
\end{aligned}
$$


Since $b_{n} a_{n}=b_{n} \delta_{n} a_{n} \delta_{n}^{-1}$, it follows from B3(iv) that $\sup _{n} b_{n} a_{n}<\infty$.

Hence it follows from B5(ii), (iii) and B3(iv) that

$$
\sum\left\{2 b_{n} \delta_{n}\left(a_{n}^{2} \alpha_{n}\left(Y_{n}\right)+1\right) Z^{2}+2 b_{n} a_{n}^{2} \delta_{n}^{3} \beta_{n}\left(Y_{n}\right)\right\}<\infty \quad \text { a.s. . }
$$

And it also follows from B3(v), (4.14) and (4.15) that

$$
\sum\left\{c_{n} \delta_{n}^{2}\left(a_{n} \alpha_{n}^{1 / 2}\left(Y_{n}\right)+1\right) Z+c_{n} a_{n} \delta_{n}^{3} \beta_{n}^{1 / 2}\left(Y_{n}\right)\right\}<\infty \quad \text { a.s. } .
$$

Hence it follows from (4.12), (4.16), (4.17) and B3(vi) that

$$
\sum \delta_{n}^{3}\left|F_{n}\left(X_{n+1}\right)-F_{n+1}\left(X_{n+1}\right)\right|_{0}\left|S_{n}\right|_{0}<\infty \quad \text { a.s. . }
$$

Then, using Kronecker lemma we obtain (c). Thus proof of lemma is complete.

Using Lemmas 2, 5 and 6 we obtain the following theorem which is the main result of this paper.

Theorem 1. Let $X_{1}, X_{2}, \cdots$ be the Robbins-Monro type process defined by (1.2).

Suppose that A1 to $\mathrm{A} 4$ and $\mathrm{B} 1$ to $\mathrm{B} 7$ are satisfied. Then it holds that

$$
\lim _{n \rightarrow \infty}\left|X_{n}-\theta_{n}\right|=0 \quad \text { a.s. . }
$$

ProOF. We reduce the theorem to an a.s. pointwise application of Lemma 2 (with $\left.u_{n}=1\right)$. Using the inequality $2 a b \leqq k a^{2}+k^{-1} b^{2}$ which holds for any $k>0$, we obtain

$$
2\left|\left\langle X_{n}-\theta_{n}, \theta_{n}-\theta_{n+1}\right\rangle\right| \leqq \frac{\alpha_{0}}{2} a_{n}\left|X_{n}-\theta_{n}\right|^{2}+2 \alpha_{0}^{-1} a_{n} A_{n},
$$

where $A_{n}$ is defined by (4.4). Let us write

$$
\left|\mathrm{E} G_{n}\left(Y_{n}\right)\right|_{0}=B_{n} .
$$

Then it follows from B3(i) that

$$
\begin{aligned}
2\left|\left\langle F_{n}\left(X_{n}\right), \mathrm{E} G_{n}\left(Y_{n}\right)\right\rangle_{0}\right| & \leqq 2 K_{1} B_{n}\left|X_{n}-\theta_{n}\right|^{2}+2 K_{2} B_{n}\left|X_{n}-\theta_{n}\right|+2 K_{3} B_{n} \\
& \leqq\left(2 K_{1} B_{n}+\frac{\alpha_{0}}{2}\right)\left|X_{n}-\theta_{n}\right|^{2}+\left(2 \alpha_{0}^{-1} K_{2}^{2} B_{n}+2 K_{3}\right) B_{n}
\end{aligned}
$$

Hence, if $\left|X_{n}-\theta_{n}\right|^{2}>\varepsilon_{n}$ then it follows from B1 that

$$
\begin{aligned}
-2 a_{n}\left\langle X_{n}-\theta_{n}, \Phi_{n}\left(X_{n}, Y_{n}\right)\right\rangle \leqq & -2 \alpha_{0} a_{n}\left|X_{n}-\theta_{n}\right|^{2}-2 a_{n}\left\langle F_{n}\left(X_{n}\right), G_{n}\left(Y_{n}\right)\right. \\
& \left.-\mathrm{E} G_{n}\left(Y_{n}\right)\right\rangle_{0}+2 a_{n}\left|\left\langle F_{n}\left(X_{n}\right), \mathrm{E} G_{n}\left(Y_{n}\right)\right\rangle_{0}\right| \\
\leqq & \left(-\frac{3}{2} \alpha_{0} a_{n}+2 K_{1} B_{n} a_{n}\right)\left|X_{n}-\theta_{n}\right|^{2} \\
& +2\left(\alpha_{0}^{-1} K_{2}^{2} B_{n}+K_{3}\right) B_{n} a_{n}+w_{n} a_{n},
\end{aligned}
$$

where

$$
w_{n}=-2\left\langle F_{n}\left(X_{n}\right), G_{n}\left(Y_{n}\right)-\mathrm{E} G_{n}\left(Y_{n}\right)\right\rangle_{0} .
$$

Substituting B5(i), (4.5), (4.19) and (4.20) into (4.2), we obtain

$$
\left|X_{n+1}-\theta_{n+1}\right|^{2} \leqq\left(1-\alpha_{0} a_{n}+2 K_{1} B_{n} a_{n}+2 a_{n}^{2} \alpha_{n}\left(Y_{n}\right)\right)\left|X_{n}-\theta_{n}\right|^{2}
$$




$$
\begin{aligned}
& +2 a_{n}^{2} \beta_{n}\left(Y_{n}\right)+a_{n} w_{n}+2 a_{n}\left(\alpha_{0}^{-1} K_{2}^{2} B_{n}+K_{3}\right) B_{n} \\
& +2 a_{n}\left(a_{n}+\alpha_{0}^{-1}\right) A_{n} \quad \text { if } \quad\left|X_{n}-\theta_{n}\right|^{2}>\varepsilon_{n} .
\end{aligned}
$$

Hence (4.21) together with (4.8) we obtain

$$
\begin{aligned}
\left|X_{n+1}-\theta_{n+1}\right| \leqq & \max \left\{h_{n},\left(1-\alpha_{0} a_{n}+2 K_{1} B_{n} a_{n}+2 a_{n}^{2} \alpha_{n}\left(Y_{n}\right)\right)\left|X_{n}-\theta_{n}\right|^{2}\right. \\
& +2 a_{n}^{2} \beta_{n}\left(Y_{n}\right)+a_{n} w_{n}+2 a_{n}\left(\alpha_{0}^{-1} K_{2}^{2} B_{n}+K_{3}\right) B_{n} \\
& \left.+2 a_{n}\left(a_{n}+\alpha_{0}^{-1}\right) A_{n}\right\}
\end{aligned}
$$

where $h_{n}$ is to be defined by (4.9). Since $\lim _{n \rightarrow \infty} B_{n}=\lim _{n \rightarrow \infty}\left|\mathrm{E} G_{n}\left(Y_{n}\right)\right|_{0}=0$, there exists a positive integer $n_{1}$ such that, for all $n \geqq n_{1}$,

$$
1-\alpha_{0} a_{n}+2 K_{1} B_{n} a_{n} \leqq 1-\frac{1}{2} \alpha_{0} a_{n} .
$$

Therefore it follows that, for all $n \geqq n_{1}$,

$$
\left|X_{n+1}-\theta_{n+1}\right|^{2} \leqq \max \left\{h_{n},\left(1-\frac{1}{2} \alpha_{0} a_{n}+d_{n}^{\prime \prime}\right)\left|X_{n}-\theta_{n}\right|^{2}+a_{n} b_{n}^{\prime \prime}+a_{n} w_{n}+v_{n}^{\prime \prime}\right.
$$

where

and

$$
d_{n}^{\prime \prime}=2 a_{n}^{2} \alpha_{n}\left(Y_{n}\right), \quad v_{n}^{\prime \prime}=2 a_{n}^{2} \beta_{n}\left(Y_{n}\right)
$$

$$
b_{n}^{\prime \prime}=2\left(\alpha_{0}^{-1} K_{2}^{2} B_{n}+K_{3}\right) B_{n}+2\left(\alpha_{0}^{-1}+a_{n}\right) A_{n} .
$$

Hence applying Lemma 2 (with $u_{n}=1$ ), it follows from (4.10), (3.1), (3.2), B7 and Lemma 6 that (4.18) holds. Thus the proof of the theorem is complete.

TheOREM 2. Let $X_{1}, X_{2}, \cdots$ be the Robbins-Monro type process defined by (1.2). Suppose that $\mathrm{A} 1, \mathrm{~A} 2, \mathrm{~A} 3^{\prime}, \mathrm{B} 7$ and $\mathrm{C} 1$ to $\mathrm{C} 3$ are satisfied. Then it holds that

$$
\lim _{n \rightarrow \infty} \mathrm{E}\left|X_{n}-\theta_{n}\right|^{2}=0 \text {. }
$$

Moreover, suppose that A4 and A5 are satisfied. Then it also hold that

$$
\lim _{n \rightarrow \infty}\left|X_{n}-\theta_{n}\right|=0 \quad \text { a.s. . }
$$

Proof. Using Lemma 2 (with $h_{n}=0$ and $u_{n}=1$ ) and Lemma 3, we can prove (4.24) by the similar arguments of the proof of Theorem 1. Hence the proof of (4.24) is omitted. We shall prove (4.23). Using the inequality $2 a b \leqq k a^{2}+k^{-1} b^{2}(k>0)$, we obtain

$$
\begin{gathered}
2\left|\left\langle X_{n}-\theta_{n}, \theta_{n}-\theta_{n+1}\right\rangle\right| \leqq \frac{1}{2} \alpha_{0} a_{n}\left|X_{n}-\theta_{n}\right|^{2}+2\left(\alpha_{0} a_{n}\right)^{-1}\left|\theta_{n}-\theta_{n+1}\right|^{2}, \\
2\left|\left\langle\Phi_{n}\left(X_{n}, Y_{n}\right), \theta_{n}-\theta_{n+1}\right\rangle\right| \leqq a_{n}\left|\Phi_{n}\left(X_{n}, Y_{n}\right)\right|^{2}+a_{n}^{-1}\left|\theta_{n}-\theta_{n+1}\right|^{2}
\end{gathered}
$$

and

$$
2\left|\left\langle X_{n}-\theta_{n}, \operatorname{E}_{n}\left(Y_{n}\right)\right\rangle\right| \leqq \frac{\alpha_{0}}{2}\left|X_{n}-\theta_{n}\right|^{2}+2 \alpha_{0}^{-1}\left|\mathrm{E} \gamma_{n}\left(Y_{n}\right)\right|^{2}
$$

Substituting (4.25) to (4.27), $\mathrm{C} 1(\mathrm{i})$ and $\mathrm{C} 2(\mathrm{i})$ into (4.2) we obtain 


$$
\begin{aligned}
\left|X_{n+1}-\theta_{n+1}\right|^{2} \leqq & \left(1-\alpha_{0} a_{n}+2 \alpha_{1} a_{n}^{2}\right)\left|X_{n}-\theta_{n}\right|^{2} \\
& +2\left\{1+\left(\alpha_{0} a_{n}\right)^{-1}\right\}\left|\theta_{n}-\theta_{n+1}\right|^{2}+2 a_{n}^{2} \beta_{n}\left(Y_{n}\right) \\
& +2 \alpha_{0}^{-1} a_{n}\left|\mathrm{E} \gamma_{n}\left(Y_{n}\right)\right|^{2}-2 a_{n}\left\langle X_{n}-\theta_{n}, \gamma_{n}\left(Y_{n}\right)\right. \\
& \left.-E \gamma_{n}\left(Y_{n}\right)\right\rangle .
\end{aligned}
$$

It is easily seen from A1 that there exists a positive integer $n_{0}$ such that, for all $n \geqq n_{0}$,

$$
1-\alpha_{0} a_{n}+2 \alpha_{1} a_{n}^{2} \leqq 1-\frac{1}{2} \alpha_{0} a_{n}
$$

Taking the expectation on the both side of (4.28) and from (4.29) we obtain

$$
\mathrm{E}\left|X_{n+1}-\theta_{n+1}\right|^{2} \leqq\left(1-\frac{1}{2} \alpha_{0} a_{n}\right) \mathrm{E}\left|X_{n}-\theta_{n}\right|^{2}+a_{n} b_{n}+a_{n} w_{n}, \quad n \geqq n_{0},
$$

where

and

$$
b_{n}=2\left(a_{n}+\alpha_{0}^{-1}\right)\left(a_{n}^{-1}\left|\theta_{n}-\theta_{n+1}\right|\right)^{2}+2 \alpha_{0}^{-1}\left|\mathrm{E} \gamma_{n}\left(Y_{n}\right)\right|^{2}+2 a_{n} \mathrm{E} \beta_{n}\left(Y_{n}\right)
$$

$$
w_{n}=2\left|\mathrm{E}\left\langle X_{n}-\theta_{n}, \gamma_{n}\left(Y_{n}\right)-\mathrm{E} \gamma_{n}\left(Y_{n}\right)\right\rangle\right| .
$$

It is easily seen from $\mathrm{A} 1, \mathrm{~B} 7, \mathrm{C} 1$ (ii) and $\mathrm{C} 2$ (ii) that

$$
\lim _{n \rightarrow \infty} b_{n}=0 .
$$

Hence, from (4.31) and according to Lemma 2.3 of [6], in order to show (4.23), we have only to prove that

$$
\lim _{n \rightarrow \infty} w_{n}=0
$$

First we shall prove that there exists a positive constant $K$ such that, for all $n \geqq 1$,

$$
\mathrm{E}\left|X_{n}-\theta_{n}\right|^{2} \leqq \delta_{n}^{-1} K
$$

Substituting

$$
w_{n} \leqq \frac{1}{4} \alpha_{0} \mathrm{E}\left|X_{n}-\theta_{n}\right|^{2}+4 \alpha_{0}^{-1} \mathrm{E}\left|\gamma_{n}\left(Y_{n}\right)-\mathrm{E} \gamma_{n}\left(Y_{n}\right)\right|^{2}
$$

into (4.30), it follows that, for all $n \geqq n_{0}$,

$$
\begin{aligned}
\delta_{n+1} \mathrm{E}\left|X_{n+1}-\theta_{n+1}\right|^{2} \leqq & \left(1-\frac{1}{4} \alpha_{0} a_{n}\right) \delta_{n} \mathrm{E}\left|X_{n}-\theta_{n}\right|^{2}+\delta_{n} a_{n} b_{n} \\
& +4 \alpha_{0}^{-1} a_{n} \delta_{n} \mathrm{E}\left|\gamma_{n}\left(Y_{n}\right)-\mathrm{E} \gamma_{n}\left(Y_{n}\right)\right|^{2} .
\end{aligned}
$$

Hence, using Lemma 2.3 of [6], it follows from (4.31), A2 and $\mathrm{Cl}(\mathrm{iii})$ that

$$
\lim _{n \rightarrow \infty} \delta_{n} \mathrm{E}\left|X_{n}-\theta_{n}\right|^{2}=0 \text {. }
$$

Thus (4.33) follows. Next we shall prove (4.32). Let us write

$$
\gamma_{n}^{\prime}\left(Y_{n}\right)=\gamma_{n}\left(Y_{n}\right)-\mathrm{E} \gamma_{n}\left(Y_{n}\right) \text {. }
$$


Then it follows from (1.2) that

$$
\begin{aligned}
\left\langle X_{n}-\theta_{n}, \gamma_{n}^{\prime}\left(Y_{n}\right)\right\rangle= & \left\langle X_{1}-\theta_{1}, \gamma_{n}^{\prime}\left(Y_{n}\right)\right\rangle+\sum_{j=1}^{n-1}\left\langle\theta_{j}-\theta_{j+1}, \gamma_{n}^{\prime}\left(Y_{n}\right)\right\rangle \\
& -\sum_{j=1}^{n-1} a_{j}\left\langle\Phi_{j}\left(X_{j}, Y_{j}\right), \gamma_{j}^{\prime}\left(Y_{j}\right)\right\rangle .
\end{aligned}
$$

Since $X_{1}$ and $\theta_{n}$ 's are constant and $\left|\mathrm{E} \gamma_{n}^{\prime}\left(Y_{n}\right)\right|=0$, it follows that

$$
\left|\mathrm{E}\left\langle X_{n}-\theta_{n}, \gamma_{n}^{\prime}\left(Y_{n}\right)\right\rangle\right| \leqq \sum_{j=1}^{n-1} a_{j}\left|\mathrm{E}\left\langle\Phi_{j}\left(X_{j}, Y_{j}\right), \gamma_{n}^{\prime}\left(Y_{n}\right)\right\rangle\right|
$$

Since $\Phi_{j}\left(X_{j}, Y_{j}\right)$ and $\gamma_{n}^{\prime}\left(Y_{n}\right)$ are measurable with respect to $\underset{1 \leq i \leq j}{\bigvee} \mathcal{A}_{i}$ and $\underset{n \leq i}{\bigvee} \mathcal{A}_{i}$ respectively, it follows from Lemma 4 and its remark that, for $1 \leqq j \leqq n-1$

$$
\begin{aligned}
& \left|\mathrm{E}\left\langle\Phi_{j}\left(X_{j}, Y_{j}\right), \gamma_{n}^{\prime}\left(Y_{n}\right)\right\rangle\right| \\
\leqq & 2 \phi^{1 / 2}\left(\underset{1 \leq i \leq j}{\bigvee} \mathcal{A}_{i}, \underset{n \leqq i}{\bigvee} \mathcal{A}_{i}\right)\left(\mathrm{E}\left|\Phi_{j}\left(X_{j}, Y_{j}\right)\right|^{2}\right)^{1 / 2} \times\left(\mathrm{E}\left|\gamma_{n}^{\prime}\left(Y_{n}\right)\right|^{2}\right)^{1 / 2} \\
\leqq & \left(\mathrm{E}\left|\Phi_{j}\left(X_{j}, Y_{j}\right)\right|^{2}+\mathrm{E}\left|\gamma_{n}^{\prime}\left(Y_{n}\right)\right|^{2}\right) \lambda_{n-j}
\end{aligned}
$$

where

$$
\lambda_{n}=\sup _{m} \phi^{1 / 2}\left(\underset{1 \leq i \leq m}{\bigvee} \mathcal{A}_{i}, \underset{n+m \leq i}{\bigvee} \AA_{i}\right), \quad n \geqq 1
$$

Substitutieg (4.35), (4.33) and C2(i) into (4.34) we obtain

$$
\begin{aligned}
\left|\mathrm{E}\left\langle X_{n}-\theta_{n}, \gamma_{n}^{\prime}\left(Y_{n}\right)\right\rangle\right| & \leqq \sum_{j=1}^{n-1} \alpha_{1} a_{j} \delta_{j}^{-1} \lambda_{n-j}+\sum_{j=1}^{n-1} a_{j} \lambda_{n-1} \mathrm{E} \beta_{j}\left(Y_{j}\right) \\
& +\sum_{j=1}^{n-1} a_{j} \lambda_{n-j} \mathrm{E}\left|\gamma_{n}^{\prime}\left(Y_{n}\right)\right|^{2} .
\end{aligned}
$$

The mixing condition $\mathrm{C} 3$ yields

$$
\sum_{j=1}^{n-1} \lambda_{n-j} \leqq \sum_{n=1}^{\infty} \lambda_{n}<\infty \text { and } \quad \lim _{n \rightarrow \infty} \lambda_{n-j}=0
$$

for any fixed $j$.

Hence, using Toeplitz lemma (see, e.g., Loéve [2], page 238), it follows from $\mathrm{A} 3^{\prime}$ and (4.36) that

$$
\lim _{n \rightarrow \infty} \sum_{j=1}^{n-1} \alpha_{1} a_{j} \delta_{j}^{-1} \lambda_{n-j}=0 .
$$

And Al, C2(ii) and (4.36) imply

$$
\lim _{n \rightarrow \infty} \sum_{j=1}^{n-1} a_{j} \lambda_{n-j} \mathrm{E} \beta_{j}\left(Y_{j}\right)=0 .
$$

Moreover, A1, C1(iii) and (4.36) imply

$$
\lim _{n \rightarrow \infty} \sum_{j=1}^{n-1} a_{j} \lambda_{n-j} \mathrm{E}\left|\gamma_{n}^{\prime}\left(Y_{n}\right)\right|^{2}=0 .
$$


Thus (4.32) is proved and the proof of the theorem is complete.

REMARK. C3 is only used to prove (4.32). In the proof of (4.24), C3 can be replaced by $\mathrm{B} 6$.

\section{Examples.}

In this section we shall give two examples of our results. The following Example 1 is the direct application of Theorem 2 .

EXAMPLE 1. Let us chose $\left\{a_{n}\right\}$ so that $a_{n}=n^{-1}$. Let us assume that $\Phi_{n}(x, y)$ can be expressed in the form of

$$
\Phi_{n}(x, y)=M_{n}(x)+\Gamma_{n}(y)
$$

where $M_{n}(\cdot)$ and $\Gamma_{n}(\cdot)$ are $\boldsymbol{R}^{N}$-balued Borel functions defined on $\boldsymbol{R}^{N}$ and $\boldsymbol{R}^{M}$, respectively. Suppose that there exist positive constants $A_{0}, A_{1}$ and $A_{2}$ such that

Moreover, suppose that

$$
\begin{aligned}
& \left\langle x-\theta_{n}, M_{n}(x)\right\rangle \geqq A_{0}\left|x-\theta_{n}\right|^{2}, \\
& \left|M_{n}(x)\right| \leqq A_{1}\left|x-\theta_{n}\right|^{2}+A_{2} .
\end{aligned}
$$

$$
\begin{aligned}
& \left|\mathrm{E} \Gamma_{n}\left(Y_{n}\right)\right|=0, \\
& \sup _{n} \mathrm{E}\left|\Gamma_{n}\left(Y_{n}\right)\right|^{2}<\infty, \\
& \lim _{n \rightarrow \infty} n\left|\theta_{n}-\theta_{n+1}\right|=0 .
\end{aligned}
$$

Let $\delta$ be a positive number satisfying $0<\delta \leqq \frac{1}{3}$. Define $\delta_{n}=n^{-\delta}$. Then A1, A2, A3 , A4 and A5 are satisfied. Define $\gamma_{n}(\cdot)=\Gamma_{n}(\cdot), \beta_{n}(\cdot)=2 A_{2}+2\left|\Gamma_{n}(\cdot)\right|^{2}, \alpha_{0}=A_{0}$ and $\alpha_{1}=2 A_{1}$. Then it is easily seen that $\mathrm{C} 1$ and $\mathrm{C} 2$ are satisfied. Moreover, it $\mathrm{C} 3$ is satisfied then it follows from Theooem 2 that (4.23) and (4.24) hold.

REMARK. If we can assume that, for all $n \geqq 2$,

$$
\left|\mathrm{E}\left[\Gamma_{n}\left(Y_{n}\right) \mid Y_{1}, \cdots, Y_{n-1}\right]\right|=0 \text { a.s. , }
$$

so that $\left\{\Gamma_{n}\left(Y_{n}\right)\right\}_{n=2}^{\infty}$ is a sequence of martingale differences, then the condition (1.3) is automatically satisfied. Hence this case is the usual Robbins-Monro stochastic approximation. But it has not been assumed in Example 1 that (5.4) holds.

Finally we shall give the following example which is an application of Theorem 1.

EXAMPLE 2. Let $\left\{a_{n}\right\}$ be chosen so that $a_{n}=n^{-1}$. Let $\delta$ be a positive number satisfying $0<\delta \leqq \frac{1}{7}$. Define $\delta_{n}=n^{-\delta}$. Then A1 to A4 are satisfied. And suppose that all vectors considered here are to be row vectors. And let us assume that $\Phi_{n}(x, y)$ can be expressed in the form of

$$
\Phi_{n}(x, y)=\left(x-\theta_{n}\right) A_{n}(y)+\Gamma_{n}(y) .
$$

where $A_{n}(y)$ is an $N \times N$-matrix whose $(i, j)$-th element is $a_{n}^{(i, j)}(y)$ which is a real valued Borel function defined on $\boldsymbol{R}^{M}$ and $\Gamma_{n}(y)=\left(\Gamma_{n}^{1}(y), \cdots, \Gamma_{n}^{N}(y)\right)$ is a $\boldsymbol{R}^{N}$-valued 
Borel function defined on $\boldsymbol{R}^{u}$. Suppose that the following conditions are satisfied.

$$
\begin{aligned}
& \left\langle x-\theta_{n},\left(x-\theta_{n}\right) A_{n}(y)\right\rangle \geqq 0 . \\
& \left\langle x-\theta_{n},\left(x-\theta_{n}\right) \mathrm{E} A_{n}\left(Y_{n}\right)\right\rangle \geqq \alpha_{0}\left|x-\theta_{n}\right|^{2}
\end{aligned}
$$

where $\alpha_{0}$ is some positive constant. And

$$
\sup _{n} \mathrm{E}\left\|A_{n}\left(Y_{n}\right)\right\|^{2}<\infty
$$

where $\|A\|$ denotes the operator norm of an $N \times N$-matrix $A$, i. e., $\|A\|=\sup _{x \mid \geq 1}|x A|$. Moreover, suppose that (5.1) to (5.3) are satisfied. Let us denote $x=\left(x^{1}, \cdots, x^{N}\right)$ and $\theta_{n}=\left(\theta_{n}^{1}, \cdots, \theta_{n}^{N}\right)$. And let us write $\left(x^{i}-\theta_{n}^{i}\right)\left(x^{j}-\theta_{n}^{j}\right)=x_{n, i, j}$ and $a_{n}^{(i, j)}(y)-\operatorname{E} a_{n}^{(i, j)}\left(Y_{n}\right)$ $=A_{n}^{(i, j)}(y)$. Define $N_{0}=N+N^{2}, \varepsilon_{n}=0, f_{n}(x, y)=\left\langle x-\theta_{n}, \Gamma_{n}(y)\right\rangle$,

$$
F_{n}(x)=\left(x_{n, 1,1}, x_{n, 1,2}, \cdots, x_{n, 2,1}, \cdots, x_{n, N, N}, x^{1}-\theta_{n}^{1}, \cdots, x^{N}-\theta_{n}^{N}\right)
$$

and

$$
G_{n}(y)=\left(A_{n}^{(1,1)}(y), A_{n}^{(1,2)}(y), \cdots, A_{n}^{(2,1)}(y), \cdots, A_{n}^{(N, N)}(y), \Gamma_{n}^{1}(y), \cdots, \Gamma_{n}^{N}(y)\right) .
$$

Since $\left\langle x-\theta_{n}, \Phi_{n}(x, y)\right\rangle=\left\langle x-\theta_{n},\left(x-\theta_{n}\right) A_{n}(y)\right\rangle+\left\langle x-\theta_{n}, \Gamma_{n}(y)\right\rangle$ and

$$
\begin{aligned}
\left\langle x-\theta_{n}, \Phi_{n}(x, y)\right\rangle= & \left\langle x-\theta_{n},\left(x-\theta_{n}\right) \mathrm{E} A_{n}\left(Y_{n}\right)\right\rangle+\left\langle x-\theta_{n},\left(x-\theta_{n}\right)\right. \\
& \left.\times\left(A_{n}(y)-\mathrm{E} A_{n}\left(Y_{n}\right)\right)+\Gamma_{n}(y)\right\rangle,
\end{aligned}
$$

(5.5) and (5.6) imply B1. And the fact $\mathrm{E}\left\|A_{n}\left(Y_{n}\right)-\mathrm{E} A_{n}\left(Y_{n}\right)\right\|^{2} \leqq 4 \mathrm{E}\left\|A_{n}\left(Y_{n}\right)\right\|^{2}$ and (5.1), (5.2), (5.3) imply B4. Note that

$$
\begin{gathered}
\left|\left\langle x-\theta_{n}, \Gamma_{n}(y)\right\rangle\right| \leqq \frac{n^{-\delta}}{2}\left|x-\theta_{n}\right|^{2}+\frac{n^{\delta}}{2}\left|\Gamma_{n}(y)\right|^{2} \\
\left|x_{n, i, j}\right| \leqq\left|x-\theta_{n}\right|^{2} \\
\left|x_{n, i, j}-x_{n, i, j}^{0}\right|=\left|\left(x^{i}-x_{0}^{i}\right)\left(x^{j}-\theta_{n}^{j}\right)+\left(x^{j}-x_{0}^{j}\right)\left(x_{0}^{i}-\theta_{n}^{i}\right)\right| \\
\leqq \\
\leqq \\
\end{gathered}
$$

and

$$
\begin{aligned}
\left|x_{n, i, j}-x_{n+1, i, j}\right|= & \mid\left(x^{i}-\theta_{n}^{i}\right)\left(\theta_{n+1}^{j}-\theta_{n}^{j}\right)+\left(x^{j}-\theta_{n}^{j}\right)\left(\theta_{n+1}^{j}-\theta_{n}^{j}\right) \\
& +\left(\theta_{n}^{i}-\theta_{n+1}^{i}\right)\left(\theta_{n}^{j}-\theta_{n+1}^{j}\right) \mid \\
\leqq & 2\left|\theta_{n}-\theta_{n+1}\right|\left|x-\theta_{n}\right|+\left|\theta_{n}-\theta_{n+1}\right|^{2} .
\end{aligned}
$$

And define $g_{n}(y)=\frac{n^{-\delta}}{2}, \quad h_{n}(y)=\frac{n^{\delta}}{2}, \quad b_{n}=0, \quad c_{n}=2\left|\theta_{n}-\theta_{n+1}\right| \quad$ and $\quad d_{n}=\left|\theta_{n}-\theta_{n+1}\right|^{2}$ $+\left|\theta_{n}-\theta_{n+1}\right|$. Then it is easily seen that B2 and B3 are satisfied. Futhermore, define $\alpha_{n}(y)=2\left\|A_{n}(y)\right\|^{2}$ and $\beta_{n}(y)=2\left|\Gamma_{n}(y)\right|^{2}$. Then (5.2) and (5.7) imply B5. Hence, if B6 is satisfied then (4.18) holds. And (5.1) and (5.6) imply that the equation (1.1) has the unique solution $x=\theta_{n}$. Futhermore, (5.7) implies

$$
\lim _{n \rightarrow \infty}\left|M_{n}\left(X_{n}\right)\right|=0 \text { a.s. , }
$$

where $M_{n}(x)=\mathrm{E} \Phi_{n}\left(x, Y_{n}\right)=\left(x-\theta_{n}\right) \mathrm{E} A_{n}\left(Y_{n}\right)$. Thus our analysis is general enough to 
include our previous result which was discussed in [7] as special case.

\section{References}

[1] Iosifescu, M. and Theodorescu, R., Random Processes and Learning, Springer, New York, 1969.

[2] LOEve, M., Probability Theory (3rd edition), Van Nostrand, New York, 1963.

[3] Robbins, H. and Monro, S., A stochastic approximation method, Ann. Math. Statist., 22 (1951), 400-407.

[4] Venter, J.H., On Dvoretzky stochastic approximation theorem, Ann. Math. Statist., 37 (1966), 1534-1544.

[5] WASAX, M.T., Stochastic Approximation, University Press, Cambridge, 1969.

[6] WataNaBe, M., On asymptotically optimal algorithms for pattern classification problems, Bull. Math. Statist., 15 (1973), 31-48.

[7] WATAxABE, M., An almost sure convergence theorem in a stochastic approximation method with dependent random variables, Bull. Math. Statist., 19 (1979), 95-112. 